\title{
Endocardite infecciosa: o que mudou na última década?
}

\author{
Infective endocarditis: what has changed in the last decade?
}

\author{
Ângelo A. Salgado* \\ Cristiane C. Lamas \\ Márcio N. Bóia
}

\section{Resumo}

A endocardite infecciosa (EI) é uma doença de alta morbidade e letalidade, apesar do avanço no diagnóstico clínico, do advento de novos tipos de antibióticos e do aperfeiçoamento de técnicas cirúrgicas. A padronização de critérios clínicos e laboratoriais de diagnóstico e a uniformização de condutas terapêuticas são estratégicas para melhorar o impacto da doença.

Um achado importante na patogenia da EI é a lesão endotelial por turbulência do fluxo sanguíneo, seja o gerado por uma valva defeituosa (reumática, valva aórtica bicúspide, prótese valvar disfuncionante), ou por anomalia congênita (comunicação interventricular, cardiomiopatia hipertrófica obstrutiva, coarctação aórtica). A presença de dispositivos intracardíacos, como marcapassos e desfibriladores implantáveis, pode servir como suporte para a fixação de trombos e vegetações. Nas EIs são acometidas mais comumente as valvas mitral (40\%) e aórtica (34\%), seguida pelo acometimento de ambas. As infecções das valvas tricúspide e/ou pulmonar ocorrem mais em usuários de drogas endovenosas e como complicação relacionada à infecção de cateter vascular profundo. A valva pulmonar é raramente acometida. As vegetações são avascularizadas, tornando o tratamento difícil pelo baixo acesso dos antibióticos aos micro-organismos, o que exige tempo de tratamento prolongado e alta concentração sérica de antibióticos administrados por via parenteral.

O ecocardiograma transtorácico (ETT) e o transesofágico (ETE) são de extrema importância para o diagnóstico, prognóstico, avaliação de complicações e para o seguimento dos pacientes com EI. Na presença de valvas degeneradas, calcificadas ou na presença de próteses mecânicas ou dispositivos intracardíacos, a utilização do ETE é de escolha para a visualização de vegetações. Propõem-se esquemas terapêuticos para as diferentes apresentações clínicas da endocardite, com base no agente etiológico provável e, quando possível, no agente isolado, utilizando-se protocolos internacionais de tratamento e adaptando-os ao contexto brasileiro. 
Enfatiza-se a importância da escolha do acesso vascular para a antibiotiocoterapia prolongada.

Descritores: Endocardite; Valvas cardíacas; Ecocardiografia; Antibacterianos.

\section{Abstract}

Infective endocarditis (IE) remains a disease with high morbidity and mortality despite advances in clinical diagnosis, in the development of new antibiotics and in improvement in surgical techniques. Standardizing clinical and laboratory diagnostic criteria and establishing treatment protocols are strategies that improve disease impact.

An important finding in the pathogenesis of IE is endothelial lesion caused by turbulent blood flow, may it be that generated by a defective valve (rheumatic valve, bicuspid aortic valve, malfunctioning valve prosthesis), by a congenital defect (interventricular septum, hypertrophic cardiomyopathy, aortic coarctation). The presence of intracardiac devices, such as pacemakers or implanted defibrillators, may serve as surfaces that promote adherence of thrombi and development of vegetations. Valves most often affected in IE are the mitral valve (40\%) and the aortic valve (34\%), followed by concomitant affection of both. Tricuspid and/ or pulmonary valve IE occurs more often in intravenous drug users and as a complication of deep venous catheter blood stream infection. The pulmonary valve is seldom affected. Vegetations are avascular structures, making treatment difficult due to the low concentrations that reach microorganisms. This demands prolonged administration and a high serum concentration of antibiotics, administered parenterally.

Transthoracic echocardiograms (TTE) and transesophageal echocardiograms (TEE) are extremely important for diagnosis, prognosis, assessment of complications and for patient follow up. In the presence of degenerative valve disease, calcification or prosthetic valves, and intravascular devices, TEE is the method of choice for best evaluation of vegetations. Tre- atment protocols are proposed for the different clinical presentations of endocarditis, based on the most likely etiological agent or the isolated microorganism, when possible. These are based on international treatment guidelines, adapted to the Brazilian scenario. Emphasis is placed on the importance of intravascular access for prolonged antibiotic treatment.

Keywords: Endocarditis; Heart valves; Echocardiography; Anti-bacterial agents.

\section{Introdução}

Entende-se por endocardite o processo inflamatório do endocárdio, sobretudo aquele localizado nas valvas cardíacas. ${ }^{1}$ Existem vários fatores causadores de endocardite, sendo o mais frequente e letal a endocardite causada por agentes infecciosos.

Apesar do avanço clínico, do advento de novos tipos de antibióticos e da progressão das técnicas cirúrgicas, a endocardite infecciosa (EI) ainda é uma doença com alta taxa de letalidade e de sequelas. ${ }^{2}$

\section{Patogênese}

O mecanismo mais comum é a lesão endotelial por turbulência do fluxo sanguíneo, seja o gerado por meio de uma valva defeituosa (reumática, valva aórtica bicúspide, prótese valvar disfuncionante), seja por alguma anomalia congênita que cause turbulência do fluxo (comunicação interventricular, cardiomiopatia hipertrófica obstrutiva, coarctação aórtica). O local mais comum de lesão e, portanto, o local mais frequente de formação de vegetação, está na linha de fechamento de uma valva, geralmente na superfície atrial das valvas atrioventriculares ou na superfície ventricular das valvas ventriculoarteriais. ${ }^{3}$ Com a lesão endotelial ocorre a formação de um trombo estéril, que pode ser colonizado por bactérias que aí se multiplicam, transformando o trombo em vegetação. Essas vegetações são avascularizadas, tornando o tratamento da endocardite difícil, pelo baixo acesso dos antibióticos aos micro-organismos, 
explicando o tempo prolongado de tratamento dessas infecções, bem como a necessidade de um diagnóstico clinicoecocardiográfico imediato. ${ }^{4}$

A presença de dispositivos intracardíacos, como cabos de marcapasso e de desfibriladores implantáveis, pode servir como suporte para fixação desses trombos e vegetações. A comprovação de infecção nesses dispositivos pode ser difícil, mesmo com a utilização de ecocardiografia transesofágica (ETE).

\section{Considerações gerais}

A endocardite infecciosa acomete mais comumente a valva mitral (40\%) ou aórtica (34\%), seguida pelo acometimento de ambas as valvas. ${ }^{5}$ $\mathrm{O}$ acometimento das valvas tricúspide e/ou pulmonar ocorre mais comumente em usuário de drogas endovenosas e em persistência de cateter de demora em veia profunda. Em 70\% dos casos a infecção é pelo S. aureus. ${ }^{6}$ A valva mais raramente acometida é a pulmonar.

$\mathrm{Na}$ associação de vegetações em valvas esquerdas e direitas, pesquisar a presença de shunt intracardíaco (CIA/CIV).

$\mathrm{O}$ diagnóstico diferencial entre vegetação com trombos/tumores/degeneração valvar fibrinosa (excrescência de Lambl), por vezes, só é possível com associação de dados clínicos e laboratoriais sugestivos de endocardite (febre, leucocitose na EI aguda, embolia periférica séptica).

Na presença de valvas degeneradas, calcificadas, ou na presença de próteses mecânicas, a utilização do ETE geralmente é de escolha para a visualização de vegetações.

O ecocardiograma transtorácico (ETT) é melhor para a avaliação de vegetações em valvas direitas. Já para a avaliação de vegetações em dispositivos intracardíacos à direita (cabos de marcapasso ou desfibriladores), deve-se preferir a associação dos métodos (ETT/ETE). ${ }^{7}$

\section{Diagnóstico}

O diagnóstico da endocardite infecciosa é baseado nos critérios de DUKE modificados ${ }^{8}$ conforme o quadro 1. Esses critérios foram adotados consensualmente pelos clínicos e nas publicações.

\section{Critérios ecocardiográficos}

O ETT e o ETE são de extrema importância para o diagnóstico, prognóstico, avaliação de complicações e para o seguimento dos pacientes com EI. A sensibilidade do ETT para evidenciar vegetações é de 40-63\%, enquanto no ETE é de 90-100\%. . O ETE é particularmente eficiente em detectar EI em próteses valvares ${ }^{10}$ ou em valvas muito calcificadas e avaliar vegetações pequenas $(<2 \mathrm{~mm})$. Os 3 achados clássicos ao ecocardiograma indicativos de endocardite são: presença de vegetações, abscessos ou de deiscência de prótese valvar. ${ }^{11}$

A vegetação pode ser definida como a presença de massa intracardíaca oscilante ou não em valva, em outra estrutura endocardíaca, em dispositivo intracardíaco, ou em área de lesão endotelial por hiperfluxo, geralmente presente em paciente febril e com leucocitose. Pode ser única ou múltiplas e acometer mais de uma valva.

Já o abscesso tem como definição área perivalvar heterogênea, espessada, com aparência ecolúcida ou ecodensa, geralmente associado ao anel aórtico. Essa área pode naturalmente drenar para os tecidos circunvizinhos através de fístula, gerando uma cavidade que se enche na sístole e se esvazia na diástole, criando assim um pseudoaneurisma. A evidência de abscesso aórtico em paciente que implantou uma prótese aórtica há pouco tempo pode ser extremamente difícil, visto que é comum observarmos edema e hematoma periprotético logo após a sua implantação, não sendo possível diferenciá-lo de um abscesso. Aqui, o diagnóstico diferencial se dará pela avaliação evolutiva da lesão, que tende a apresentar evolutivamente, caso seja um abscesso, áreas hipoecogênicas múltiplas (lagos gangrenosos e sépticos). A existência de abscesso demonstra a presença de endocardite agressiva, sendo necessária intervenção cirúrgica precoce na maioria dos casos.

A presença de deiscência valvar é caracte- 
rizada pela identificação de regurgitação perivalvar, associada ou não a vegetações na área do refluxo. Nesse contexto,a clínica é fundamental, já que a deiscência precoce no pós-operatório pode estar associada a problema de técnica cirúrgica e não necessariamente à presença de infecção. A deiscência da sutura da prótese englobando mais de $40 \%$ do anel faz com que haja um movimento em báscula da prótese, conhecida como rockingvalve, sempre associada a importante regurgitação perivalvar. Algumas definições e alterações ecocardiográficas causadas por endocardite e suas complicações são mostradas no quadro 2 .

Nos casos em que o exame inicial seja negativo, o ETT/ETE deve ser repetido após 7-10 dias, caso haja persistência dos sintomas clínicos, em situações de alta suspeita clínica de EI ou caso seja isolado nas hemoculturas bactérias ou fungos (candida) associados à EI. ${ }^{12}$ Deve-se ter atenção especial caso seja isolado S. aureus, podendo o ETT/ETE ser repetido mais precocemente, visto que esta bactéria está relacionada a alto poder de destruição valvar, formação de abscessos e alta morbidade. Exames subsequentes estão indicados apenas caso haja piora clínica, mesmo em vigência de medicação, não sendo indicada a repetição apenas para avaliação da vegetação (quadro 3 ). ${ }^{13}$

\section{Dispositivos intracardíacos}

A infecção de dispositivos intracardíacos está associada à alta morbidade e mortalidade, ocorrendo óbito em até $18 \%$ dos casos mesmo

\section{Quadro 1. Diagnóstico de endocardite infecciosa, critérios de DUKE modificado}

\begin{tabular}{|c|}
\hline Critérios maiores \\
\hline Hemoculturas positivas: \\
\hline $\begin{array}{l}\rightarrow \text { Organismos típicos cultivados em } 2 \text { hemoculturas diferentes: Streptococcus do grupo viridans, S. aureus, } \\
\text { HACEK (Haemophilus, Actinobacillus, Cardiobacterium, Eikenella, ou Kingella), ou Streptococcus bovis; } \\
\text { Enterococcus adquiridos em comunidade na ausência de uma fonte primária de infecção; }\end{array}$ \\
\hline $\begin{array}{l}\rightarrow \text { Hemoculturas persistentemente positivas com outros organismos: } 2 \text { hemoculturas positivas com mais de } 12 \\
\text { horas de intervalo entre elas; ou positividade em todas de } 3 \text { ou a maioria de } 4 \text {, com intervalo entre a primeira e } \\
\text { última coleta maior que } 1 \text { hora; ou }\end{array}$ \\
\hline$\rightarrow$ Cultura, teste de biologia molecular ou sorologia IgG fase 1 > 1:800 para Coxiella burnetii. \\
\hline Evidência de envolvimento endocárdico: \\
\hline $\begin{array}{l}\rightarrow \text { Ecocardiograma demonstrando massa intracardíaca oscilante sem outra explicação ou abscesso, ou nova } \\
\text { deiscência parcial de uma valva protética, ou nova regurgitação valvar. }\end{array}$ \\
\hline Critérios menores \\
\hline Predisposição à El: \\
\hline $\begin{array}{l}\rightarrow \text { El prévia, uso de droga injetável, valva cardíaca protética, ou lesão cardíaca causando fluxo sanguíneo } \\
\text { turbulento. }\end{array}$ \\
\hline Febre acima de $38^{\circ} \mathrm{C}$. \\
\hline Fenômeno vascular: \\
\hline $\begin{array}{l}\rightarrow \text { Embolismo arterial, infarto pulmonar, aneurisma micótico, hemorragia intracraniana ou conjuntival, ou lesões } \\
\text { de Janeway. }\end{array}$ \\
\hline Fenômeno imunológico: \\
\hline$\rightarrow$ Glomerulonefrite, nódulos de Osler, manchas de Roth, fator reumatoide positivo. \\
\hline Achados microbiológicos que não preenchem os critérios maiores. \\
\hline $\begin{array}{l}\text { OBS: O DIAGNÓSTICO DEFINITIVO DE EI REQUER } 2 \text { CRITÉRIOS MAIORES, OU } 1 \text { MAIOR E } 3 \text { MENORES. EI PROVÁVEL REQUER } 1 \\
\text { CRITÉRIO MAIOR E } 1 \text { CRITÉRIO MENOR OU } 3 \text { CRITÉRIOS MENORES. }\end{array}$ \\
\hline
\end{tabular}

Fonte: Adaptado de Li JS, Sexton DJ, Mick N, Nettles R, Fowler VG, Jr., Ryan T, Bashore T, Corey GR. Proposed modifications to the Duke criteria for the diagnosis of infective endocarditis. Clin Infect Dis 2000;30:633-638. 
Quadro 2. Alterações ecocardiográficas na endocardite infecciosa e suas complicações

\begin{tabular}{|c|c|c|}
\hline & Cirurgia/Necropsia & Ecocardiografia \\
\hline Vegetação & $\begin{array}{l}\text { Massagem endocárdio ou em } \\
\text { dispositivo intracardíaco. }\end{array}$ & $\begin{array}{l}\text { Massa intracardíaca pedunculada em valva } \\
\text { ou em outra estrutura endocárdica ou em } \\
\text { dispositivo intracardíaco. }\end{array}$ \\
\hline Abscesso & $\begin{array}{l}\text { Cavidade perivalvar com necrose } \\
\text { ou material purulento, sem } \\
\text { comunicação com o lúmen } \\
\text { cardíaco. }\end{array}$ & $\begin{array}{l}\text { Área perivalvar heterogênea, espessada, } \\
\text { com aparência ecolúcida ou ecodensa. }\end{array}$ \\
\hline Pseudoaneurisma & $\begin{array}{l}\text { Cavidade perivalvar com } \\
\text { comunicação com o lúmen } \\
\text { cardíaco. }\end{array}$ & $\begin{array}{l}\text { Espaço perivalvar com pulsação e com } \\
\text { fluxo no seu interior, ao Color Doppler. }\end{array}$ \\
\hline Perfuração & $\begin{array}{l}\text { Interrupção da continuidade do } \\
\text { tecido endocárdico. }\end{array}$ & $\begin{array}{l}\text { Interrupção da continuidade do tecido } \\
\text { endocárdico, evidenciada ao Color } \\
\text { Doppler. }\end{array}$ \\
\hline Fístula & $\begin{array}{l}\text { Comunicação entre duas cavidades } \\
\text { contíguas através de uma } \\
\text { perfuração. }\end{array}$ & $\begin{array}{l}\text { Comunicação entre } 2 \text { cavidades contíguas } \\
\text { através de uma perfuração, evidenciada ao } \\
\text { Color Doppler. }\end{array}$ \\
\hline Aneurisma valvar & $\begin{array}{l}\text { Estrutura sacular oriunda do } \\
\text { tecido valvar. }\end{array}$ & $\begin{array}{l}\text { Estrutura sacular oriunda do tecido } \\
\text { valvar evidenciada ao ecocardiograma } \\
\text { bidimensional. }\end{array}$ \\
\hline Deiscência de prótese valvar & Deiscência de prótese. & $\begin{array}{l}\text { Regurgitação paravalvar identificada ao } \\
\text { ETT ou ao ETE com ou sem movimento de } \\
\text { báscula (rocking) da prótese. }\end{array}$ \\
\hline
\end{tabular}

Fonte: Adaptado do Guidelines on the prevention, diagnosis, and treatment of infective endocarditis (new version 2009). The Task Force on the Prevention, Diagnosis, and Treatment of Infective Endocarditis of the European Society of Cardiology (ESC). European Heart Journal 2009;30:2369-2413.

após antibioticoterapia adequada e retirada cirúrgica do dispositivo infectado. ${ }^{14}$ Ocorrem, sobretudo, por infecção por S. aureus e por várias espécies de estafilococos coagulase - negativo. A presença de 2 ou mais cabos intracardíacos (marcapasso dupla câmara e ressincronizador) aumenta a possibilidade de endocardite, bem como o reposicionamento de cabos de CDI. ${ }^{15}$ O diagnóstico, por vezes, pode ser dificultado pela presença de trombos e/ou fímbrias aderidas ao longo do cabo, não sendo possível diferenciá-las da vegetação da endocardite, havendo necessidade de acompanhamento seriado (o crescimento da massa em curto espaço de tempo sugere vegetação) e associação com outros sinais de infecção. O ETE pode ser utilizado associado ao ETT para melhor visualização de toda a extensão dos cabos, permitindo melhor acurácia diagnóstica.

\section{Complicações}

\section{Embolia}

O risco de embolia é maior logo após o diagnóstico, diminuindo significativamente após o início do tratamento antimicrobiano (sobretudo após 15 dias). Ocorre em $20 \%$ a 50\% dos casos. ${ }^{16}$ As vegetações com maior caráter emboligênico são pedunculadas, aderidas ao folheto anterior mitral e maiores de $10 \mathrm{~mm} .{ }^{17}$ Vegetações menores de $15 \mathrm{~mm}$, sobretudo causadas por S. aureus e acometendo a valva mitral, são as associadas a maior risco de embolização. ${ }^{18}$
Abscesso
Ocorre em $10 \%$ a $40 \%$ dos casos, acome- tendo valvas ou próteses aórticas e estando comumente associado ao S. aureus. ${ }^{19}$ Pela proximidade com o nó atrioventricular, pode haver evolução para bloqueio atrioventricular 
Quadro 3. Recomendações para realização de ecocardiograma transtorácico (ETT) e ecocardiograma transesofágico (ETE) nas endocardites infecciosas

\begin{tabular}{|c|c|c|}
\hline Recomendações: ecocardiografia & Classe & Nível \\
\hline \multicolumn{3}{|l|}{ A. No diagnóstico: } \\
\hline ETT é recomendado como a exame de primeira linha em caso de suspeita de El; & 1 & B \\
\hline ETE é recomendado em pacientes com alta suspeita clínica de El e ETT normal; & I & B \\
\hline $\begin{array}{l}\text { Repetição dos esquemas ETT/ETE no intervalo de 7-10 dias são recomendados no } \\
\text { caso de exame inicial negativo quando a suspeita clínica do El permanecer alta; }\end{array}$ & 1 & B \\
\hline $\begin{array}{l}\text { ETE dever ser considerado para a maioria dos pacientes adultos com suspeita } \\
\text { de El, mesmo nos casos com ETT positivo devido a sua melhor sensibilidade e } \\
\text { especificidade, particularmente para o diagnóstico de abscesso e medida da } \\
\text { dimensão da vegetação. }\end{array}$ & Ila & $\mathrm{C}$ \\
\hline $\begin{array}{l}\text { ETE não é indicado para pacientes sem evidência de EI, em ETT de ótima qualidade, } \\
\text { com baixa suspeita clínica de IE. }\end{array}$ & III & C \\
\hline \multicolumn{3}{|l|}{ B. Após o início da terapia medicamentosa } \\
\hline $\begin{array}{l}\text { 1. Novos exames pelo ETT e ETE são recomendados em suspeita de complicação da } \\
\text { EI (novo sopro, embolia, febre persistente, ICC, abscesso, bloqueio atrioventricular); }\end{array}$ & I & B \\
\hline $\begin{array}{l}\text { 2. Novos exames pelo ETT e ETE podem ser realizados durante terapêutica de El não } \\
\text { complicada, para detectar nova complicação subclínica e monitorar a dimensão } \\
\text { da vegetação. O tempo e modo (ETT/ETE) do exame depende do diagnóstico inicial, } \\
\text { tipo de micro-organismo, e resposta inicial de terapia. }\end{array}$ & Ila & B \\
\hline \multicolumn{3}{|l|}{ C. Ecocardiograma intraoperatório } \\
\hline $\begin{array}{l}\text { Ecocardiograma intraoperatório é recomendado em toda a cirurgia de El que requer } \\
\text { cirurgia. }\end{array}$ & I & $\mathrm{C}$ \\
\hline \multicolumn{3}{|l|}{ D. Após término da terapia com antibióticos } \\
\hline $\begin{array}{l}\text { ETT é recomendado ao término da antibioticoterapia para avaliação da morfologia } \\
\text { cardíaca e valvar e função do VE. }\end{array}$ & 1 & C \\
\hline
\end{tabular}

Fonte: Adaptado do Guidelines on the prevention, diagnosis, and treatment of infective endocarditis (new version 2009). The Task Force on the Prevention, Diagnosis, and Treatment of Infective Endocarditis of the European Society of Cardiology (ESC). European Heart Journal. 2009;30:2369-2413.

total (BAVT), sendo necessária a sua resolução por via cirúrgica, pela pouca penetração de antibiótico na área do abscesso.

\section{Insuficiência cardíaca}

A insuficiência cardíaca está associada à destruição valvar ou protética, sobretudo à regurgitação aórtica ou mitral graves, agudas ou subagudas, ${ }^{20}$ por ruptura de folhetos, cordoalhas ou perfurações valvares, fístulas intracardíacas e, mais raramente, pela obstrução do orifício valvar pela vegetação. Geralmente não há falência contrátil miocárdica. É considerada uma emergência cirúrgica.

\section{Tratamento}

Há várias publicações com recomendações para tratamento da endocardite. O proposto a seguir se baseia na reflexão após leitura de Baddour et al., ${ }^{11}$ Habib et al., ${ }^{21}$ Cosgrove et al. ${ }^{22}$ e mais recentemente de Gould et al. ${ }^{23}$

Antimicrobianos em endocardite são usados por via endovenosa de 4 a 6 semanas na maioria das situações, tornando a questão do acesso vascular fundamental desde o início. É recomendável o uso cateter de acesso profundo de inserção periférica (PICC), por ser o menos associado a eventos adversos infecciosos. ${ }^{24}$

Esquemas antimicrobianos em
diferentes situações clínicas

I. Endocardite infecciosa subaguda de válvula nativa e de válvula protética com mais de $\mathbf{1}$ ano de inserção 
Subaguda é definida como aquela em que sinais e sintomas tiveram início há mais de duas semanas.

Tratamento empírico:

Ampicilina $2 \mathrm{~g}$ EV 4/4 horas + gentamicina $2 \mathrm{mg} / \mathrm{kg}$ em dose única diária.

Observações:

É importante que se pese o paciente para a prescrição da dose correta de gentamicina. Deve-se usar o peso real do paciente, exceto se ele tiver índice de massa corporal (IMC) maior que 30, quando o peso ideal deverá ser usado. $\mathrm{Na}$ prescrição, a gentamicina deve ser administrada em solução endovenosa em cerca de 40 minutos, para evitar eventos adversos como paralisia respiratória. Os níveis séricos pré e pós-dose de gentamicina devem ser determinados após a terceira dose administrada, especialmente em pacientes com comorbidades como hipertensão arterial, diabetes, uso de anti-inflamatórios não esteroides ou com idade superior a 60 anos. ${ }^{11,23}$ Os valores de referência para níveis pré e pós-dose de gentamicina são respectivamente menor que $1 \mathrm{mcg} / \mathrm{ml}$ e 3 a $5 \mathrm{mcg} / \mathrm{ml}$. Os níveis devem ser monitorados uma vez por semana em doentes estáveis clínica e hemodinamicamente, e duas vezes por semana nos não estáveis. $\mathrm{O}$ racional desse esquema é prover cobertura para estreptococos do grupo viridans, enterococos e gram-negativos do grupo HACEK. Também há menor flebotoxicidade da ampicilina em relação à penicilina cristalina. Caso seja confirmado estreptococo viridans, manter esquema com ampicilina e gentamicina (por duas semanas em dose única diária); caso haja disfunção renal, a gentamicina pode ser suspensa antes. Consultar o infectologista ou microbiologista quanto ao MIC para penicilina, que pode dispensar mais cedo o uso de gentamicina.

Orientações subsequentes:

Se o paciente não for candidato cirúrgico, $o$ esquema será mudado para ceftriaxona $2 \mathrm{~g} \mathrm{EV}$ uma vez ao dia. A gentamicina será mantida por duas semanas, em dose única diária, podendo ser suspensa caso haja disfunção renal ou o MIC do isolado seja baixo para penicilina.

Se o paciente for candidato cirúrgico, sugerese manter ampicilina e gentamicina, evitando o uso de ceftriaxona precocemente, com indução de betalactamases com possível disseminação em unidades fechadas. No pós-operatório não complicado, ou na volta para a enfermaria, sugere-se substituir ampicilina por ceftriaxona $2 \mathrm{~g}$ ao dia, até se completar o tempo de tratamento.

Caso seja confirmado enterococo, manter ampicilina e fracionar a gentamicina em 3 doses diárias (1,7 mg/kg/dose). Monitorar os níveis séricos como sugerido acima. Nos casos em que o paciente tem grande risco de piora da função renal, recomenda-se a associação ampilicina com ceftriaxona $2 \mathrm{~g}$ de $12 \mathrm{em} 12$ horas para o enterococo. ${ }^{25}$ Essa associação mostrou eficácia semelhante no tratamento de EI por enterococo quando comparada à associação clássica ampicilina/gentamicina nessa publicação recente.

Caso o paciente já tenha usado antibiótico e as hemoculturas sejam negativas, o esquema ampicilina com gentamicina será mantido, uma vez que estreptococo do grupo viridans será o agente mais provável, mas enterococo é também possível agente etiológico.

Caso o paciente não tenha usado antimicrobianos antes da coleta e as hemoculturas sejam negativas em 7 dias, mudar o esquema para ceftriaxona $2 \mathrm{~g} \mathrm{EV/dia} \mathrm{(6} \mathrm{semanas)} \mathrm{+} \mathrm{gen-}$ tamicina $3 \mathrm{mg} / \mathrm{kg} /$ dia em 3 doses fracionadas (2 semanas) + doxiciclina $100 \mathrm{mg} \mathrm{VO}$ de $12 \mathrm{em}$ 12 horas (6 semanas), se a história epidemiológica for altamente sugestiva para infecção para Coxiella burnetii.

Racional:

Bartonella spp e HACEK são agentes etiológicos prováveis nessa situação.

\section{Endocardite infecciosa aguda em válvula nativa}

Definida como aquela em que sinais e sintomas tiveram início há menos de duas semanas. Tratamento empírico:

Oxacilina 2 g EV 4/4 horas (dose/kg) + vancomicina $15 \mathrm{mg}$ a $20 \mathrm{mg} / \mathrm{kg} \mathrm{12/12h}$. 
Importante:

Pesar o paciente para cálculo correto, pois a dose de vancomicina é calculada sobre o peso real dos pacientes. ${ }^{26}$ Caso obeso, recomenda-se fracionar as doses de 6 em 6 ou 8 em 8 horas, não ultrapassando a dose diária de $4 \mathrm{~g} /$ dia pelo risco muito aumentado de nefrotoxicidade nesta situação. ${ }^{27}$ A primeira dose de vancomicina será de $20 \mathrm{mg} / \mathrm{kg} .^{28} \mathrm{O}$ ajuste posterior da dose será feito após dosagem sérica de vancomicina e pelo clearance de creatinina do paciente.

Racional:

Cobertura de S. aureus sensível e não sensível a oxacilina (MRSA) da comunidade

Orientação subsequente:

- Suspensão de vancomicina após 48 horas de hemoculturas negativas ou se S. aureus isolado for sensível.

- Caso MRSA isolado, suspender oxacilina, mantendo vancomicina. Dosar níveis séricos de vancomina no terceiro dia pós-início e duas vezes por semana no decorrer do tratamento. $^{29}$

- Caso seja isolado MRSA e haja insuficiência renal moderada a grave (clearance de creatinina $<50 \mathrm{ml} / \mathrm{min}$ ), hipersensibilidade a vancomicina ou MIC da vancomicina $>1,5$ para vancomicina, usar daptomicina $6 \mathrm{mg} /$ $\mathrm{kg}$ EV mais rifampicina ou gentamicina (consultar infectologista para avaliar em conjunto).

\section{Endocardite infecciosa aguda em válvula protética}

Tratamento empírico caso manifestações clínicas ocorram com menos de 2 meses de inserção da prótese:

Vancomicina 15 mg/kg/dose de 12 em 12 horas + gentamicina $2 \mathrm{mg} / \mathrm{kg} / \mathrm{dia}$ em dose única diária + meropenem $2 \mathrm{~g} \mathrm{EV} 8 \mathrm{em} 8$ horas. Acrescentar após 5 dias rifampicina $300 \mathrm{mg}$ VO de 8 em 8 horas.
Tratamento empírico, caso endocardite de prótese com mais de 2 meses e menos de 1 ano de inserção:

Vancomicina $15 \mathrm{mg} / \mathrm{kg} /$ dose de $12 \mathrm{em} 12$ horas + gentamicina $3 \mathrm{mg} / \mathrm{kg} /$ dia em 3 doses fracionadas. Acrescentar após 5 dias rifampicina $300 \mathrm{mg}$ VO 8 em 8 horas.

Importante:

Pesar o paciente. A primeira dose de vancomicina será de $20 \mathrm{mg} / \mathrm{kg}$. Após a terceira, dosar vancomicina sérica conforme protocolo de vancocinemia.

Racional:

Cobertura para estafilococo coagulase negativo, MRSA e enterobactérias produtoras de betalactamase de espectro estendido (ESBL) quando a inserção se deu há menos de 2 meses. O início da rifampicina após 5 dias é para minimizar o risco de aquisição de resistência à mesma por estafilococos.

Consultar o infectologista para ajuste terapêutico posterior quando do isolamento de micro-organismos (preferência por betalactâmico associado à aminoglicosídeo será considerada para gram-negativos sensíveis).

Caso as culturas sejam negativas, manter vancomicina e rifampicina e consultar o infectologista quanto à cobertura para fungos.

\section{Tempo de antimicrobianos no pós-operatório}

Regras gerais a seguir:

1. Resultados da cultura da válvula devem ser buscados, mas sua sensibilidade é baixa, inferior a 30\%. Também a incidência de falsos positivos não é desprezível. Se houver crescimento de micro-organismo como da hemocultura, fazer 6 semanas de antimicrobianos pós-operatório.

2. Utilizar, como padrão-ouro, a histopatologia da valva/prótese, que deve ser buscada na semana seguinte à troca valvar.

a) Se a histopatologia mostrar endocardite infecciosa em atividade (presença de neutrófilos) com presença de bactérias, o tempo de antimicrobianos será 4 semanas pós-operatórios. 
b) Se mostrar EI em atividade sem presença de bactérias, tempo de antimicrobianos será em torno de 2 semanas.

c) Se os achados cirúrgicos mostrarem abscesso perivalvar, desinserção, perfuração de válvula nativa ou de prótese, comunicação entre cavidades (fístulas), o tempo será de 6 semanas.

d) Se a história da endocardite tem tempo de evolução superior a 6 meses, tratar por 6 semanas.

e) Se o histopatológico mostrar endocardite resolvida, manter 2 semanas de antimicrobianos pós-operatórios.

\section{Referências}

1. Que YA, Haefliger JA, Piroth L, Francois P, Widmer E, Entenza JM, et al. Fibrinogenandfibronectinbindingcooperate for valveinfectionandinvasion in Staphylococcus aureus experimental endocarditis. J Exp Med. 2005;201:1627-1635.

2. Moreillon P, Que YA. Infective endocarditis. Lancet. 2004;363:139-149.

3. Bashore TM, Cabell C, Fowler VJ Jr. Update on infective endocarditis. CurrProblCardiol. 2006;4:274-352.

4. McDonald JR. Acute Infective Endocarditis. Infect Dis Clin N Am. 2009;23:643-664.

5. Miro JM, Anguera I, Cabell CH, Chen AY, Stafford JA,Corey GR, et al. Staphylococcus aureus native valve infective endocarditis: report of 566 episodes from the International Collaboration on Endocarditis Merged Database. Clin Infect Dis. 2005;41:507-14.

6. Moss R, Munt B. Injection drug use and right sided endocarditis. Heart. 2003;89:577-81.

7. Sampedro MF, Patel Robin. Infections Associated with Long-Term Prosthetic Devices. Infect Dis Clin N Am. 2007;21:785-819.

8. Li JS, Sexton DJ, Mick N, et al. Proposed modifications to the Duke criteria for the diagnosis of infective endocarditis.Clin Infect Dis. 2000;30:633-8.

9. Evangelista A, Gonzalez-Alujas MT. Echocardiography in infective endocarditis. Heart. 2004;90:614-617.

10. Daniel WG, Mugge A, Grote J, Hausmann D, Nikutta P, Laas J, Lichtlen PR, Martin RP. Comparison of transthoracic and transesophageal echocardiography for detection of abnormalities of prosthetic and bioprosthetic valves in the mitral and aortic positions. Am J Cardiol. 1993;71:210-215.
11. Baddour LM, Wilson WR, Bayer AS, Fowler VG, Bolger AF, Levison ME, et al. Infective Endocarditis: Diagnosis, Antimicrobial Therapy, and Management of Complications: A Statement for Healthcare Professionals From the Committee on Rheumatic Fever, Endocarditis, and Kawasaki Disease, Council on Cardiovascular Disease in the Young, and the Councils on Clinical Cardiology Stroke, and Cardiovascular Surgery and Anesthesia, American Heart Association: Endorsed by the Infectious Diseases Society of America. Circulation. 2005;111:394-434.

12. Rohmann S, Erbel R, Darius H, Gorge G, Makowski T, Zotz R, et al. Prediction of rapid versus prolonged healing of infective endocarditis by monitoring vegetation size. J AmSocEchocardiogr. 1991;4:465-474.

13. Vieira ML, Grinberg M, Pomerantzeff PM, Andrade JL, Mansur AJ. Repeated echocardiographic examinations of patients with suspected infective endocarditis. Heart. 2004;90:1020-1024.

14. Klug D, Lacroix D, Savoye C, Goullard L, Grandmougin D, Hennequin JL, et al. Systemic infection related to endocarditis on pacemaker leads: Clinical presentation and management. Circulation. 1997;95:2098-2107.

15. Nery PB, Fernandes R, Nair GM, Sumner GL, Ribas CS, Menon SMD, et al. Device-Related Infection Among Patients With Pacemakers and Implantable Defibrillators: Incidence, Risk Factors, and Consequences. J Cardiovasc Electrophysiol. 2010;21:786-790.

16. Thuny F, Disalvo G, Belliard O, Averinos JF, Pergola V, et al. Risk of embolism and death in infective endocarditis: prognostic value of echocardiography, a prospective multicenter study. Circulation. 2005;112:69-75.

17. Mylonakis E, Calderwood SB. Infective endocarditis in adults. N Engl J Med. 2001;345:1318-30.

18. Vilacosta I, Graupner C, San Roman JA, Sarria C, Ronderos R, Fernandez C, et al. Risk of embolization after institution of antibiotic therapy for infective endocarditis. J Am Coll Cardiol. 2002;39:1489-1495.

19. Fernicola DJ, Roberts WC. Frequency of ring abscess and cuspal infection in active infective endocarditis involving bioprostheticvalves. Am J Cardiol. 1993;72:314-323.

20. Durack DT, Lukes AS, Bright DK. New criteria for diagnosis of infective endocarditis: utilization of specific echocardiographic findings. Duke Endocarditis Service. Am J Med. 1994;96:200-209.

21. Habib G, Hoen B, Tornos P, Thuny F, Prendergast B, Vilacosta I, et al. Guidelines on the prevention, diagnosis and treatment of infective endocarditis new version 2009. European Heart Journal. 2009;30:2369-2413.

22. Cosgrove SE, Vigliani GA, Campion M, Fowler VG Jr, Abrutyn E, Corey RG, et al. Initial low- 
dose gentamicin for Staphylococcus aureus bacteremia and endocarditis is nephrotoxic. Clin Infect Dis 2009;48:713-21.

23. Gould FK, Elliott TSJ, Foweraker J, Fulford M, Perry JD, Roberts GJ, et al. Guidelines for the diagnosis and antibiotic treatment of endocarditis in adults: a report of the Working Party of the British Society for Antimicrobial Chemotherapy. J Antimicrob Chemother. 2012;67:269-289.

24. De Paula DHG, Tura B, Lamas C. Adverse events related to intravenous antibiotic therapy: a prospective observational study in the treatment of infective endocarditis. BMJ Open. 2012;2:e001189.

25. Fernandez-Hidalgo N, Almirante B, Gavaldà J, Gurgui M, Peña C, de Alarcón A, et al. Ampicillin plus ceftriaxone is as effective as ampicillin plus gentamicin for treating Enterococcus faecalis infective endocarditis. ClinInfec Dis. 2013 May;56(9):1261-8. http:// dx.doi.org/10.1093/cid/cit052.

26. JansonB ,Thursky K. Dosing of antibiotics in obesity. Curr Opinion Infect Dis. 2012;25:634649.

27. Lodise TP, Lomaestro B, Graves J, Rodvold KA, Drusano GL. Larger vancomycin doses (at least four grams per day) are associated with an increased incidence of nephrotoxicity. Antimicrob Agents Chemother. 2008;52:13301336.

28. Liu C, Bayer E, Cosgrove SE, Daum RS, Fridkin SK, Gorwitz RJ, et al. Clinical Practice Guidelines by the Infectious Diseases Society of America for the treatment of Methicillinresistant Staphylococcus aureus in adults and children. Clin Infect Dis. 2011:1-38.

29. Rybak M, Lomaestr BM, Rotscahfer JC, Moellering RC, Craig WA, Billeter M, et al Vancomycin therapeutic guideline: a summary consensus recommendations from the Infectious Diseases Society of America, the American Society of Health-System Pharmacist, and the Society of Infectious Diseases Pharmacists. Clin Infect Dis. 2009;49:325-7. 